\title{
Copd and Periodontitis ... Past Facts and the Present Status!!!
}

\author{
Dr. Sri Varshini Poojitha Senapathi, BDS ${ }^{1}$, Dr. Reema Lakshmanan, MDS ${ }^{2}$ \\ ${ }^{1}$ (Saveetha Dental College/ Saveetha University, India) \\ ${ }^{2}$ (Department of Periodontics, Saveetha Dental College / Saveetha University, India)
}

\begin{abstract}
Periodontitis is a chronic bacterial infection characterized by persistent inflammation, connective tissue breakdown and alveolar bone destruction. Accumulating evidence suggests periodontitis and its possible link in the development of respiratory infections like bacterial pneumonia and chronic obstructive pulmonary disease (COPD). COPD is characterized by blockage in the airflow and difficulty in breathing .The etiological factors of COPD are smoking and tobacoo, however, periodontopathic bacteria and genetic factors also play an important role in the pathogenesis. Routes of infection occur by aspiration of oral pathogens in to the lungs, colonization of dental plaque by respiratory pathogens followed by aspiration. The periodontopathic bacteria is also known to facilitate the subsequent colonization of respiratory tract by pulmonary pathogens. This review throws light on the pathogenesis and available evidences on the relationship between periodontitis and COPD.
\end{abstract}

Keywords: COPD, Periodontitis, Respiratory pathogens, Periodontal Pathogens, Inflammatory mediators.

\section{Introduction}

Chronic periodontitis is a destructive disease affecting the tooth supporting tissues. Its course tends to be progressive, although bursts can occur and it is associated with imbalance between subgingival plaque, mainly consisting of gram negative bacteria and host defence [1].The relationship between periodontal health or disease and systemic health or disease has given rise to a discipline in periodontology termed "Periodontal Medicine" aptly termed and proposed by Offenbacher at 1996 World Wokshop in Periodontics[2].

Out of the various systemic diseases, an area of particular interest is the link between COPD (Chronic Obstructive Periodontal Disease) with periodontitis . COPD is characterized by chronic blockage in the airflow and difficulty in breathing and includes chronic bronchitis, emphysema and sometimes asthma[3].

The main etiological factor is tobacco smoking but bacteria may play a key role in the progression of the disease. Genetic factors have also been strongly implicated in the pathogenesis[4].

Pneumonia (both community acquired and hospital acquired) is an acute infection of the lung characterized by cough, shortness of breath, sputum production and chest pain. It is caused by the micro aspiration of the oropharyngeal secretions containing bacteria into the lung and failure of the host to clear out the bacteria. Community acquired pneumonia is typically caused by aspiration of bacteria that reside normally in the oropharynx such as streptococcus pneumonia, haemophilus influenza and mycoplasm pneumonia. In contrast, nosocomial pneumonia is usually caused by bacteria that normally do not colonise the oropharynx , including species such as Pseudomonas aerugnosa, staphylococcus aureus and gram negative bacteria such as klebsilla pneumonia, escherichia coli and enterobacter species[5].

This review discusses the relationship between COPD and oral health and the underlying pathogenic mechanisms.

\section{Risk factors for COPD}

Respiratory disease are the diseases of the respiratory system that range from common cold to life threatening diseases like asthma, COPD and pneumonia [6].COPD is one of the most common respiratory diseases and is projected to be the fourth leading cause of death worldwide by 2030 and places a huge economic burden on society[7]. The disease is characterized by airflow limitation that is not fully reversible, which is caused by a combination of obstructive bronchiolitis (also known as chronic bronchitis) and destruction of alveoli resulting in airspace enlargement (also known as emphysema)[8].The main risk factor for COPD is smoking, however, air pollutants, respiratory infections, genetic factors and hereditary are among other risk factors of COPD[3].

\section{Inflammatory cells and mediators in COPD}

Cigarette smoke triggers an inflammatory response in the airway epithelium of the proximal and distal bronchi, in the alveoli, and in the pulmonary arteries[9].Recruitment of inflammatory cells in the airways and alveoli involves the secretion of chemokines by resident cells (eg, epithelial cells and alveolar macrophages) in response to cigarette smoke[10].Cigarette smoke and chronic infections also lead to the activation of multiple 
Pathogen Recognition Receptors contributing to mucus hyper secretion by epithelial cells, release of proteases by recruited neutrophils and fibroblast proliferation[11,12]. Destruction of alveolar walls (emphysema) occurs in COPD as a result of protease-mediated degradation of connective tissue elements, particularly elastin, and apoptosis of pneumocytes and endothelial cells[13]. Proinflammatory cytokines, such as TNF- $\alpha$, IL-1 $\beta$, and IL6 , are increased in COPD, and appear to amplify inflammation, in part through the activation of the transcription factor, nuclear factor NF-k $\beta$, thereby leading to the increased expression of multiple inflammatory genes[10]. A striking characteristic of the chronic inflammation in COPD lungs and its failure to resolve on stopping smoking, even after several years, suggest that some endogenous mechanism, such as autoimmunity or persistent systemic infection, may be driving the inflammatory process[11,14].

\section{Relationship between periodontal infection and COPD}

In recent years a possible association between COPD and periodontitis have been arrived at. Oral cavity being continuous with the trachea serve as a possible portal entry for the colonisation of respiratory pathogens in to the lower respiratory tract as a result of oropharyngeal aspiration[15]. Scannapeico and Ho et al in 2001 showed an association between COPD and oral health based on the analysis of existing database , National Health and Nutrition Examination Survey III(NHANES III) after controlling for confounding variables like smoking, sex, age and socioeconomic status[16].

A systematic review by Azarpazhooh and Leake in 2006 , consisting of 728 papers discussed the possibility of an etiological association between respiratory disease and oral health. He concluded that there is a fair evidence of an association of pneumonia with oral health but there is a poor evidence of a weak association between COPD and oral health[17].

\section{Mechanism of action}

There are two routes for oral microorganism to reach the lower respiratory tract: hematogenous spread, aspiration and inhalation. Hematogenous spread as a result of bacteriemia is an adverse effect in most dental procedure and may occur after some prophylactic procedure such as scaling[18]. Scannapeico in 1999 described four modes by which oral pathogens play a role in the pathogenesis of respiratory infections. Oral pathogens such as P.ginivalis and Aggregaribacter actinomycetemcomitans are aspirated into the lungs. It is conceivable that in subjects having periodontal disease and elevated levels of proteolytic bacteria such as P.gingivalis and spirochetes, protease activity may alter the mucosal epithelium to increase the adhesion and colonisation of respiratory pathogens. An other mechanism is by the destruction of protective salivary pellicles by oral bacteria. Thus an increased microbial load may result in elevated levels of salivary hydrolytic enzymes , which in turn destroy protective barriers. This diminishes the non specific host defence against respiratory pathogens in high risk subjects[15]. Lastly the epithelial cells are also known to alter the expression of various cell adhesion molecules on their surface in response to cytokine stimulation. Variation in expression of such adhesion molecules may alter the interaction of bacterial pathogens with the mucosal surface[19].

Inhalation of air borne pathogens can cause pulmonary infection. Dental plaque also serve as a reservoir for respiratory pathogens. They colonise the subgingival areas and are also shed into saliva. This saliva contaminates the respiratory tract resulting in pulmonary infection[15].

\section{Respiratory and periodontal pathogens}

A variety of oral anaerobes and facultative species have been cultured from the infected lungs such as P.gingivalis, Fusobacterium nucleatum, Fusobacterium necrophorum, Bacteriodes oralis, Aggregatibacter actinomycetemcomitans [20,21] that can cause both periodontal disease and respiratory infection [22].

\section{Recent Studies}

A recent workshop was conducted in 2013 by European Federation of Periodontology and American Academy of Periodontology based on the NHANES data sets .The workshop conducted was to known relationship between periodontitis and various systemic diseases. There appears to be a dose effect, whereby greater periodontal disease is associated with increasing loss of lung function. The primary etiological factor is smoking as modified by underlying inflammation .It is plausible that the inflammatory status may be modified either by aspiration of dental plaque and /or hematogenous dissemination of inflammatory mediators and plaque organisms from periodontal pockets .Studies of the association between periodontal disease and exacerbations of COPD would be valuable. The authors also concluded that newer studies should fulfill the Bradford Hill or equivalent criteria. Respiratory diseases under consideration are also complex and multifactorial in nature. They were all in agreement that studies should also focus on diseases outcomes rather than focusing only on the biomarkers[23]. 


\section{Conclusion}

The available evidence for COPD and its relationship in periodontitis is at an immature level. This opens the door to carry out further clinical trials to provide quality evidence.

\section{References:}

[1] Page RC, Offenbacher S, Schroeder HE, Seymour GJ, Kornman KS , Advances in the pathogenesis of periodontitis: summary of developments, clinical implications and future directions, Periodontol 2000,14,1997, 216-48.

[2] Williams RC, Offenbacher S, Periodontal medicine: the emergence of a new branch of periodontology. Periodontol 2000, 23, 2000, 9-12.

[3] American Lung Association, Chronic Obstructive Pulmonary Disease (COPD), Available at: http;//www.lungusa.org/site/pp.asp

[4] Stanford AJ,Weir TD, Pare PD,Genetic risk factors for chronic obstructive pulmonary disease, Eur Resp J , 10, 1997,1380-1391.

[5] Bartlett JG, Bacteriology of hospital acquired pneumonia, Arch Itern Med, 146(5), 1986, 868-71.

[6] Weidlich P, Cimões R, Pannuti CM, Oppermann RV, Association between periodontal diseases and systemic diseases. Braz Oral Res, 22 (1), 2008, 32-43.

[7] Mathers CD, Loncar D, Projections of global mortality and burden of disease from 2002 to 2030. PLoS Med, 3(11), 2006 , e442.

[8] J. E. McDonough, R. Yuan, M. Suzuki et al, Small-airway obstruction and emphysema in chronic obstructive pulmonary disease ,The New England Journal of Medicine,367 (17), 2011, 1567-1575.

[9] Burgel PR, Bourdin A, Chanez P, et al . Update on the roles of distal airways in COPD. Eur Respir Rev, $20,2011,7-22$.

[10] Barnes PJ. The cytokine network in chronic obstructive pulmonary disease. Am J Respir Cell Mol Biol, 41, 2009, 631-638 .

[11] Cosio MG, Saetta M, Agusti A. Immunologic aspects of chronic obstructive pulmonary disease. $N$ Engl J Med, 360, 2009, 24452454 .

[12] Hansel TT, Barnes PJ. New drugs for exacerbations of chronic obstructive pulmonary disease. Lancet ,374, 2009, 744-755.

[13] Majo J, Ghezzo H, Cosio MG. Lymphocyte population and apoptosis in the lungs of smokers and their relation to emphysema. Eur Respir J, 17, 2001, 946-953.

[14] Taraseviciene-Stewart L, Douglas IS, Nana-Sinkam PS, Lee JD, Tuder RM, Nicolls MR, Voelkel NF. Is alveolar destruction and emphysema in chronic obstructive pulmonary disease an immune disease? Proc Am Thorac Soc , 3, 2006, 687-690.

[15] Scannapieco FA , Role of Oral Bacteria in Respiratory Infection, J Periodontol 70, 1999, 793-802.

[16] Scannapieco FA, Ho AW, Potential associations between chronic respiratory disease and periodontal disease: analysis of National Health and Nutrition Examination Survey III. J Periodontol,72(1), 2001, 50-6 .

[17] Azarpazhooh A, Leake JL, Systematic review of the association between respiratory diseases and oral health. J Periodontol, 77(9),2006, 1465-82

[18] S.Anil,The Emerging Concept On The Impact Of Periodontitis On Systemic Health . Jane Manakil(Editior) "Periodontal Disease A clinicians guide" (Intech Publishers, Feb 3 2012).

[19] Svanborg C, Hedlund M, Connell H et al .Bacterial adherence and mucosal cytokines responses. Receptors and transmembrane signaling, Ann N Y Acad Sci,797, 1996, 177-190.

[20] Brook I, Frazier EH. Aerobic and anearobic microbiology of empyema, A retrospective review in two military hospitals, Chest, 103,1993, 1502-1507.

[21] Zijilstra EE, Swart GR, Godfroy FJ, Degener JE ,Pericarditis, pneumonia and brain abscess due to a combined Actinomycesactinobacillus actinomycetemcomitans infection , $J$ infect , 25, 1992, 83-87.

[22] Moore WE, Moor LV ,The bacteria of periodontal diseases, Periodontol 2000, 15, 1994, 66-77.

[23] Linden JG,Herzberg MC, Periodontitis and systemic diseases:a record of discussion of working group 4 of the Joint EFP/AAP Workshop on Periodontitis and Systemic Diseases , J Periodontol , 84(4 suppl.), 2013, S20-3. 